\title{
Effects of Semi-purified Dietary Fibers Isolated from Lagenaria siceraria, Raphanus sativus and Lentinus edodes on Fecal Steroid Excretions in Rats
}

\author{
Yasuko Sannoumaru, ${ }^{*}$ Jun Shimizu, Kahoru NaKamura, ${ }^{1}$ \\ Takashi HAYAKAwa, ${ }^{2}$ Toshichika TaKita, \\ and Satoshi INNAMI** \\ Department of Nutrition, Faculty of Agriculture, Tokyo University \\ of Agriculture, Setagaya-ku, Tokyo 156, Japan \\ ${ }^{1}$ Department of Nutrition, Junior College of Agriculture, Tokyo University \\ of Agriculture, Setagaya-ku, Tokyo 156, Japan \\ ${ }^{2}$ Department of Utilization of Biological Resources, Gifu University, \\ Gifu 501-11, Japan
}

(Received August 12, 1995)

Summary Rats were fed diets, each of which contained 5\% of the semi-purified dietary fiber (DF) preparations from three kinds of foods, continuously for a total of 7 weeks. The fat level was varied, and cholesterol (Chol) was added toward the end of the experimental period. After 7 weeks, decreases of serum and liver Chol concentrations were observed in the Shiitake (Lentinus edodes) group when compared to the cellulose (CP) group. In the absence of Chol, fecal excretions of several secondary bile acids and total bile acids were affected by the type of DF, showing an increase particularly in the Shiitake group. Total neutral steroid excretions were decreased in the test DF groups compared to the $\mathrm{CP}$ group at both fat levels, and were increased by Chol supplementation in all of the test DF groups. It was demonstrated that the effects of the three DF preparations on fecal steroid excretions somewhat differed depending on the dietary factors. In the absence of Chol, the lithocholic acid (LCA)/deoxycholic acid (DCA) ratio was significantly lowered in the test DF groups compared to the CP group by the fat level elevation, and was affected by the interaction of fiber with fat. This ratio responded more intensely to the dietary factors than the two other indices (composition ratios of fecal bile acids). However, this response was lost in the presence of Chol. These results suggest that further studies are warranted to examine if the intense response of the LCA/DCA ratio to the tested DF preparations and fat in the present study will also be shown to other DF

\footnotetext{
* Present address: The Skylark Food Science Institute, Chiba 261, Japan

${ }^{* *}$ To whom correspondence should be addressed.
} 
and what significance it has in evaluating the function of DF.

Key Words semi-purified dietary fibers, fecal steroid excretion, bile acids, neutral sterols, lithocholic acid/deoxycholic acid ratio

Since epidemiological studies by Reddy and Wynder $(1,2)$ and Hill (3) demonstrated that there was a significant positive correlation between the fecal bile acid (BA) concentration and the incidence of colon cancer, BA metabolism in the intestine has been a matter of growing concern. A large number of subsequent animal experiments (4-6) and in vitro studies (7-9) suggest that cholesterol (Chol) or BA, or their metabolites, act as co-carcinogenic or co-mutagenic promoters. Not only the total excretion of BA but also the type of BA and the ratio of secondary BA to total BA have therefore been considered important. Subsequently, Wilpart and Roberfroid (9) and Owen et al. $(10,11)$ hypothesized that the ratio of lithocholic acid (LCA) to deoxycholic acid (DCA) in feces is a more sensitive index for the risk of colon cancer.

The quality and quantity of lipids (12-14) and the type and quantity of dietary fibers (DF) (15-17) are known to be the dietary factors which modify fecal BA excretion. The quantity of dietary Chol is also a potent factor $(18,19)$. The effects of DF on the composition of fecal BA differ depending on the type of DF and experimental conditions, and do not always show similar spectra. In recent years, attention has been directed toward the interaction of dietary fats and DF (20), leading to studies on their effects on BA metabolism $(21,22)$. However, their results are not necessarily concordant because of the effect of the type of DF, and the data on this subject are therefore scarce.

In the present study, we selected the semipurified preparations extracted from three kinds of foods which are popular DF sources in Japan and have not yet fully been studied regarding variations of fecal steroid profiles. Then, we carried out experiments in succession in order to investigate what effects the increase of dietary fats would have on the response of fecal steroids in rats fed DF preparations with different hemicellulose composition and how the addition of Chol to the diet would modify the composition of fecal steroids under DF influence.

\section{MATERIALS AND METHODS}

Preparation and composition of DF. The DF samples used in the study were obtained from the following foods: 1) Kanpyo (dried white flower gourd, Lagenaria siceraria STANDLEY var. hispicda HARA) (produce of Tochigi Prefecture, Suyama Co., Ltd.), 2) Kiriboshi daikon (Kiriboshi, dried Japanese radish strips, Raphanus sativus L.) (produce of Miyagi Prefecture, Yamaki Y Co., Ltd.) and 3) Shiitake, dried (a kind of fungus, Lentinus edodes (BERK) Sing.) (produce of Ohita Prefecture, Yamada Shoten Co., Ltd.). The samples were soaked in water for $30 \mathrm{~min}$, squeezed lightly and cut into fine strips (hard tips of Shiitake stems were 
removed). The cut samples were freeze-dried in a vacuum, powdered with a coffee mill and passed through a 30-mesh sieve. DF was extracted from the powdered samples by the modified AOAC enzymatic gravimetric method (23). The alcohol precipitates were filtered through a Buchner funnel, washed with alcohol and acetone, and air-dried. General components of the samples were routinely determined and the DF compositions were analyzed according to the Southgate method (24). The results are shown in Table 1, indicating semi-purified preparations. There were no marked differences in the DF content of roughly $85 \%$ among the three samples although there were some differences in hemicellulose fraction.

Animals, diet composition and rearing method. Male Sprague-Dawley strain rats aged 4 weeks (Clea Japan, Inc., Tokyo) were used in the study. They were housed individually in stainless steel cages. The animal room was maintained at $22 \pm 1{ }^{\circ} \mathrm{C}, 55 \pm 5 \%$ humidity, and a light/dark cycle of $12 \mathrm{~h}$ (lighting: 8:00-20:00). After rats were fed the AIN-76 ${ }^{\mathrm{TM}}$ basal diet modified with lard instead of corn oil for one week, those without any abnormality in growth were divided into four groups of six rats each, and were given the experimental diets. The basal diet (control) was composed of 25\% casein, 5\% lard, 49.8\% sucrose, 3.5\% mineral mixture $\left(\mathrm{AIN}-76^{\mathrm{TM}}\right), 1.0 \%$ vitamin mixture $\left(\mathrm{AIN}-76^{\mathrm{TM}}\right), 0.2 \%$ choline bitartrate and $5 \%$ cellulose.

The DF preparations were added at the level of 5\% to each experimental diet. Rats were fed the diets for a total of 7 weeks, with 5\% lard in the first 3 weeks, $10 \%$ lard for the following 2 weeks and 10\% lard and $0.5 \%$ of Chol in the last 2 weeks. The differences arising from alteration in the dietary ingredients in the experimental period (4th to 7 th weeks) were adjusted with sucrose.

During the 7-week experimental period, feces were collected for the last 5 days in the $3 \mathrm{rd}, 5$ th and 7 th weeks and weighed before and after vacuum freeze-drying to determine the water content. Feces were powdered and kept at $-30^{\circ} \mathrm{C}$ until analysis. The animals were starved for $7 \mathrm{~h}$ from $6: 00$ to $13: 00$ on the last day of the experiment and sacrificed by cardiocentesis under pentobarbital anesthesia. The organs including the digestive tract were weighed, and serum was obtained. The liver was perfused with ice-cooled physiological saline injected under pressure from the portal vein to remove blood and weighed, and $0.5 \mathrm{~g}$ of the tissue was sampled for

Table 1. Composition of semi-purified dietary fiber samples (\%).

\begin{tabular}{lccc}
\hline & Kanpyo & Kiriboshi & Shiitake \\
\hline Moisture & 3.2 & 3.8 & 3.6 \\
Protein & 9.7 & 6.0 & 7.2 \\
Ash & 3.2 & 4.5 & 0.9 \\
Total fiber & 83.9 & 85.7 & 88.3 \\
$\quad$ Water soluble hemicellulose & 9.7 & 3.0 & 2.7 \\
Water insoluble hemicellulose & 22.6 & 29.3 & 36.9 \\
Cellulose & 50.0 & 51.1 & 45.9 \\
Lignin & 1.6 & 2.3 & 2.7 \\
\hline
\end{tabular}


extracting lipids with a chloroform : methanol $(2: 1)$ mixture on the day of sacrifice.

Measurements of serum and liver lipids and fecal steroids. Serum total cholesterol (TC), triglycerides (TG) and phospholipids (PL) and liver TG and PL were determined using the kits available from Wako Pure Chemical Industries, Ltd. (Osaka, Japan), and liver TC by the Zak method (25).

Fecal BA and neutral sterols (NS) were measured by gas chromatography according to the method of Grundy et al. (26). Extracted fecal lipids were enzymatically deconjugated and saponified to separate them into BA and NS fractions. They were then subjected to methylation and trifluoroacetylation using an acetyl chloride: methanol $(1: 20)$ mixture and trifluoroacetic anhydride (TFA; Wako Pure Chemical) for use as analytical samples. The internal standard substances used for BA and NS analyses were respectively nor-deoxycholic acid (3,12-dihydroxy-nor-cholanic acid) synthesized by the method of Riegel et al. (27) and cholestanone (Sigma Chemical Co., MO, USA). A Shimadzu GC 12A (Shimadzu Co., Kyoto, Japan) attached with a flame ionization detector was used for gas chromatography. The analytical conditions were as follows: BA: column, $1.5 \%$ AN-600 on 100-120 mesh Gaschrom Q (1.6 m $\times 3 \mathrm{~mm}$ ID, Japan Chromato Industry, Tokyo); column temperature, $225^{\circ} \mathrm{C}$; injector and detector temperature, $250^{\circ} \mathrm{C}$; carrier gas, $\mathrm{N}_{2}$ (flow rate, $65 \mathrm{ml} / \mathrm{min}$ ); NS: column, $3 \% \mathrm{SP}-2401$ on $100-120$ mesh Supelcoport $(1.6 \mathrm{~m} \times 2.6 \mathrm{~mm}$ ID, Supelco, PA, USA); column temperature, $220^{\circ} \mathrm{C}$; injector and detector temperature, $250^{\circ} \mathrm{C}$; carrier gas, $\mathrm{N}_{2}$ (flow rate, 35 $\mathrm{ml} / \mathrm{min}$ ).

Statistical processing. Individual group comparisons were made using Duncan's new multiple range test $(p<0.05)$. Data were also analyzed by two-way ANOVA using the SAS computer package. A separate analysis was carried out to determine the significance of fiber, fat or fiber $\times$ fat (weeks 3 and 5), and fiber, Chol or fiber $\times$ Chol (weeks 5 and 7). This procedure was adopted to minimize the influence of aging as much as possible.

\section{RESULTS}

Body weight gain, food intake, liver weight, and serum and liver lipid concentrations

The results are shown in Table 2. Hardly any intergroup differences were observed in body weight gain, food intake and liver weight for the entire feeding period. Serum TC and PL concentrations were generally high in the Kanpyo group and low in the Kiriboshi and Shiitake groups when compared to the CP group. The values for the Kiriboshi and Shiitake groups were significantly lower than those in the Kanpyo group. Liver TC concentrations in the Shiitake group were significantly lower than those of the CP group as well as the other two DF groups.

\section{Fecal weight and water content}

The results are shown in Table 3. Compared to the CP group, the fecal wet and dry weights showed significant decreases in all the measurement weeks in all of 
Table 2. Body weight gain, food intake, liver weight and serum and liver lipid concentrations in rats fed on the experimental diets for 7 weeks. ${ }^{1}$

\begin{tabular}{lcccc}
\hline \multicolumn{1}{c}{ Items } & Cellulose & Kanpyo & Kiriboshi & Shiitake \\
\hline Body weight gain (g/day) & $6.33 \pm 0.22$ & $6.27 \pm 0.16$ & $5.73 \pm 0.24$ & $5.92 \pm 0.14$ \\
Food intake (g/day) & $20.9 \pm 0.41$ & $20.5 \pm 0.31$ & $19.6 \pm 0.78$ & $20.1 \pm 0.49$ \\
Liver (g) & $18.8 \pm 0.98$ & $19.7 \pm 0.92$ & $17.1 \pm 0.92$ & $17.9 \pm 0.74$ \\
Serum (mg/dl) & & & & \\
$\quad$ Total cholesterol & $129 \pm 11.9^{\mathrm{ab}}$ & $160 \pm 14.9^{\mathrm{a}}$ & $111 \pm 4.6^{\mathrm{b}}$ & $104 \pm 8.6^{\mathrm{b}}$ \\
$\quad$ Triglycerides & $347 \pm 78.7$ & $443 \pm 57.8$ & $288 \pm 37.8$ & $306 \pm 40.8$ \\
$\quad$ Phospholipids & $199 \pm 20.6^{\mathrm{ab}}$ & $215 \pm 13.3^{\mathrm{a}}$ & $168 \pm 8.8^{\mathrm{b}}$ & $168 \pm 12.5^{\mathrm{b}}$ \\
Liver (mg/g) & & & & \\
$\quad$ Total cholesterol & $19.0 \pm 1.2^{\mathrm{a}}$ & $24.2 \pm 2.5^{\mathrm{a}}$ & $22.8 \pm 2.2^{\mathrm{a}}$ & $13.7 \pm 0.7^{\mathrm{b}}$ \\
$\quad$ Triglycerides & $64.9 \pm 4.8^{\mathrm{a}}$ & $70.0 \pm 4.7^{\mathrm{ab}}$ & $87.8 \pm 9.1^{\mathrm{b}}$ & $85.6 \pm 8.1^{\mathrm{ab}}$ \\
$\quad$ Phospholipids & $31.0 \pm 1.5^{\mathrm{a}}$ & $33.2 \pm 1.7^{\mathrm{ab}}$ & $36.9 \pm 1.0^{\mathrm{b}}$ & $31.8 \pm 1.4^{\mathrm{a}}$ \\
\hline
\end{tabular}

${ }^{1} \mathrm{M} \pm \operatorname{SEM}(n=6)$ : means in a same line with different superscript letter are significantly different $(p<0.05)$.

the DF groups, but the fecal water content showed a significant increase in all week in the Kanpyo and Shiitake groups. The fat level affected only the fecal dry weight while Chol addition affected the fecal weight as well as the fecal water content. Fat, however, affected neither these parameters nor the interaction of Chol addition and DF.

\section{Fecal steroid excretions}

Tables 4 and 5 show the changes in fecal BA excretions in rats fed the experimental diets. In the absence of Chol, the different types of DF produced differences in the excretions of BA per $1 \mathrm{~g}$ of feces excluding CA and $\omega$-muricholic acid ( $\omega$-MCA), the excretions in the Shiitake group being particularly increased. The analysis of BA excretions per day in the absence of Chol revealed that DCA was strongly affected by the type of DF and the fat level while total BA showed the effect of the type of DF but not of the fat level. Chol addition significantly elevated the concentration of each BA as well as that of total BA and increased the excretion per day in all the groups. Intergroup differences were observed except in the LCA concentration. However, no interaction was observed between fiber and fat or fiber and Chol (excluding $\omega$-MCA).

Table 6 shows the relation between DF and the ratios of BA. The secondary/total BA ratio was affected by fiber but more intensely by the fat level. Chol addition exerted no effect. There was no interaction between fiber and fat or between fiber and Chol. The CDCA/CA ratio was lowered in the DF groups compared to the CP group and with elevation in the fat level. Chol addition raised the ratio in the DF groups. The LCA/DCA ratio was also decreased in the DF groups compared to the CP group at either of the fat levels, and the decrease was also produced by elevation in the fat level. In contrast, Chol addition raised the 


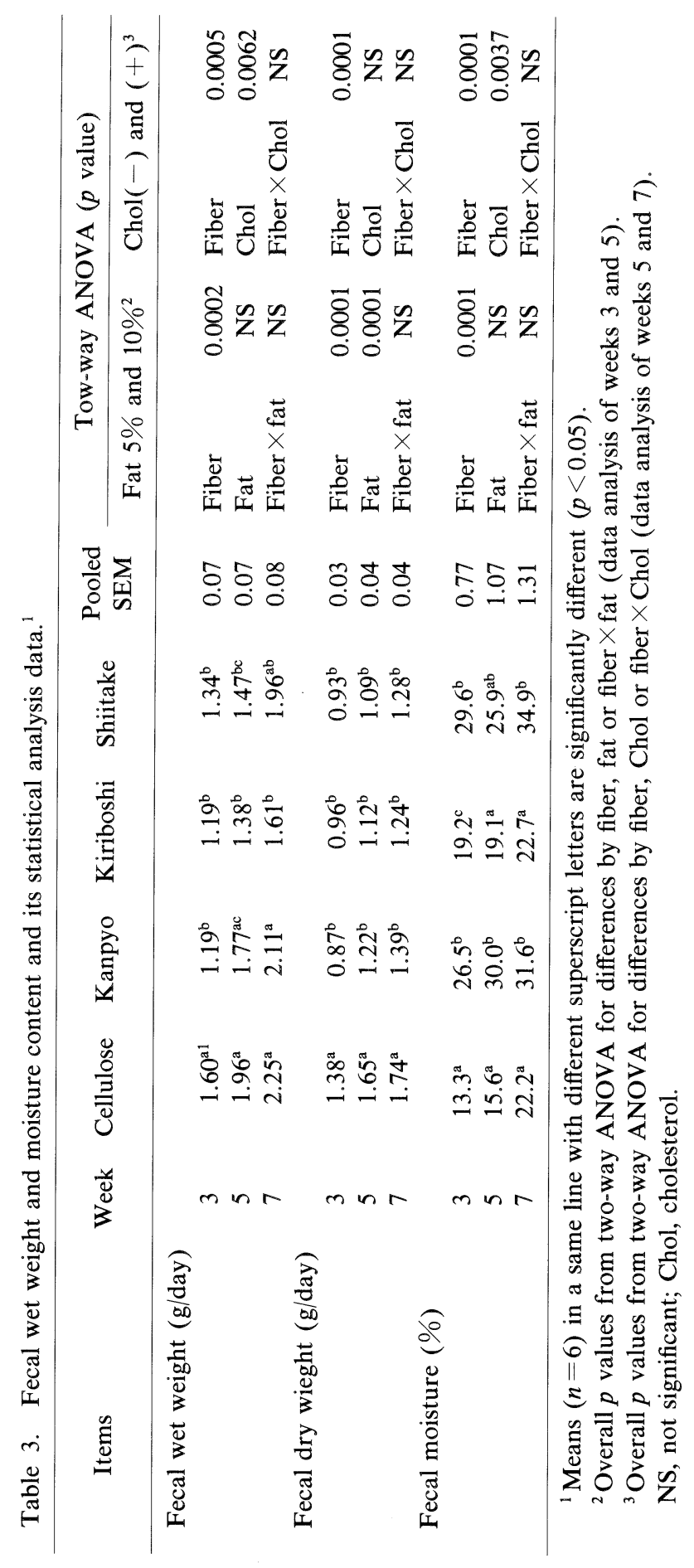




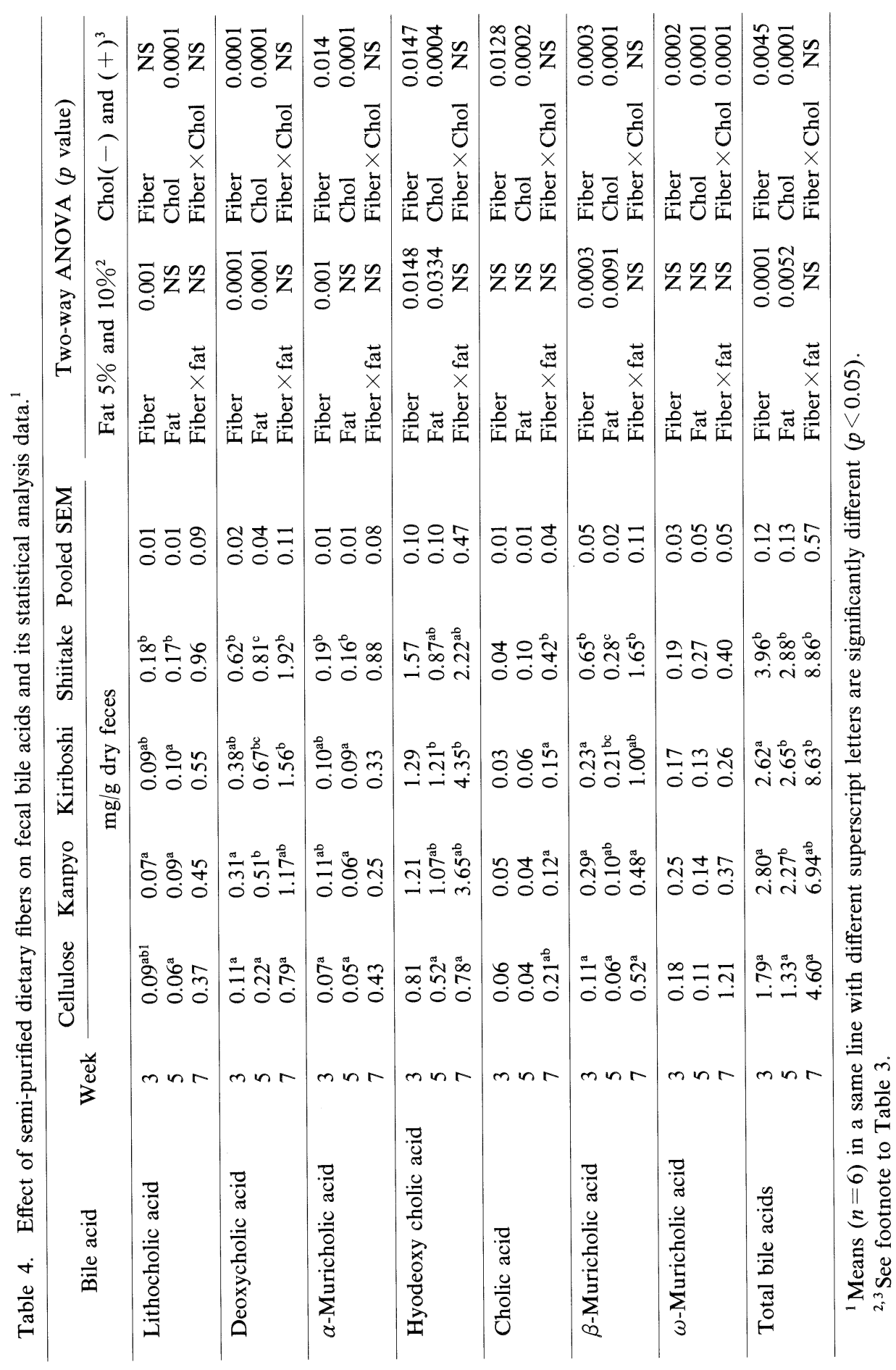

Vol. 42, No. 2, 1996 


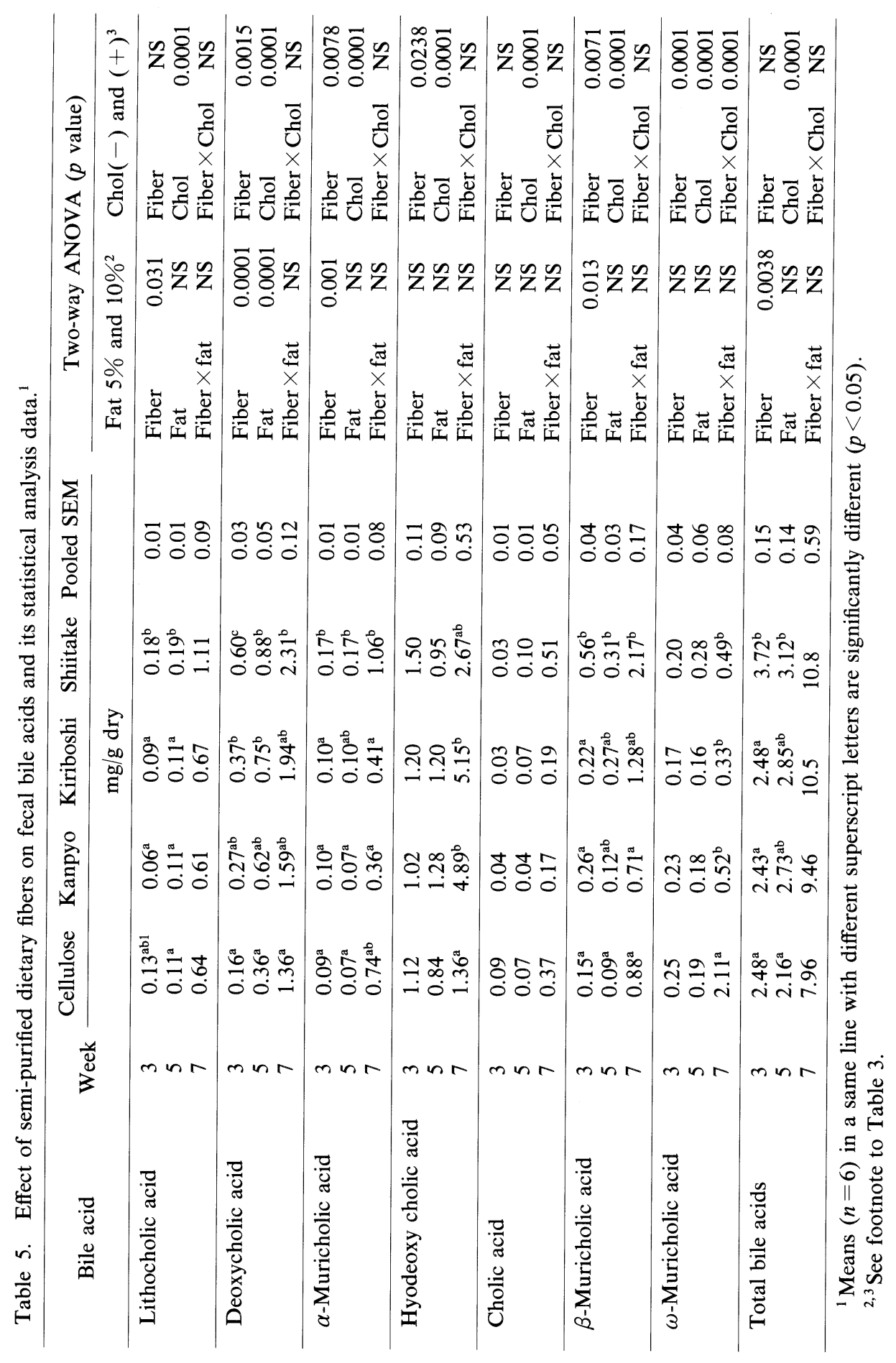




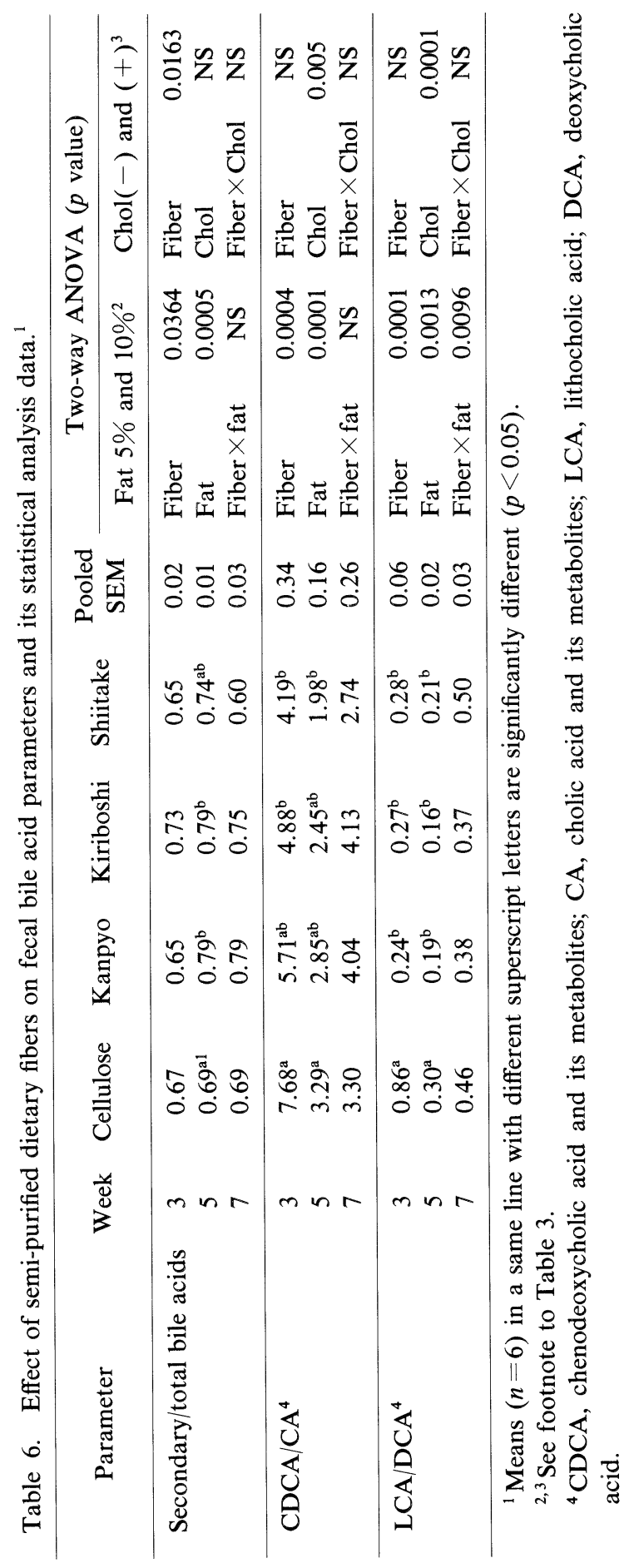




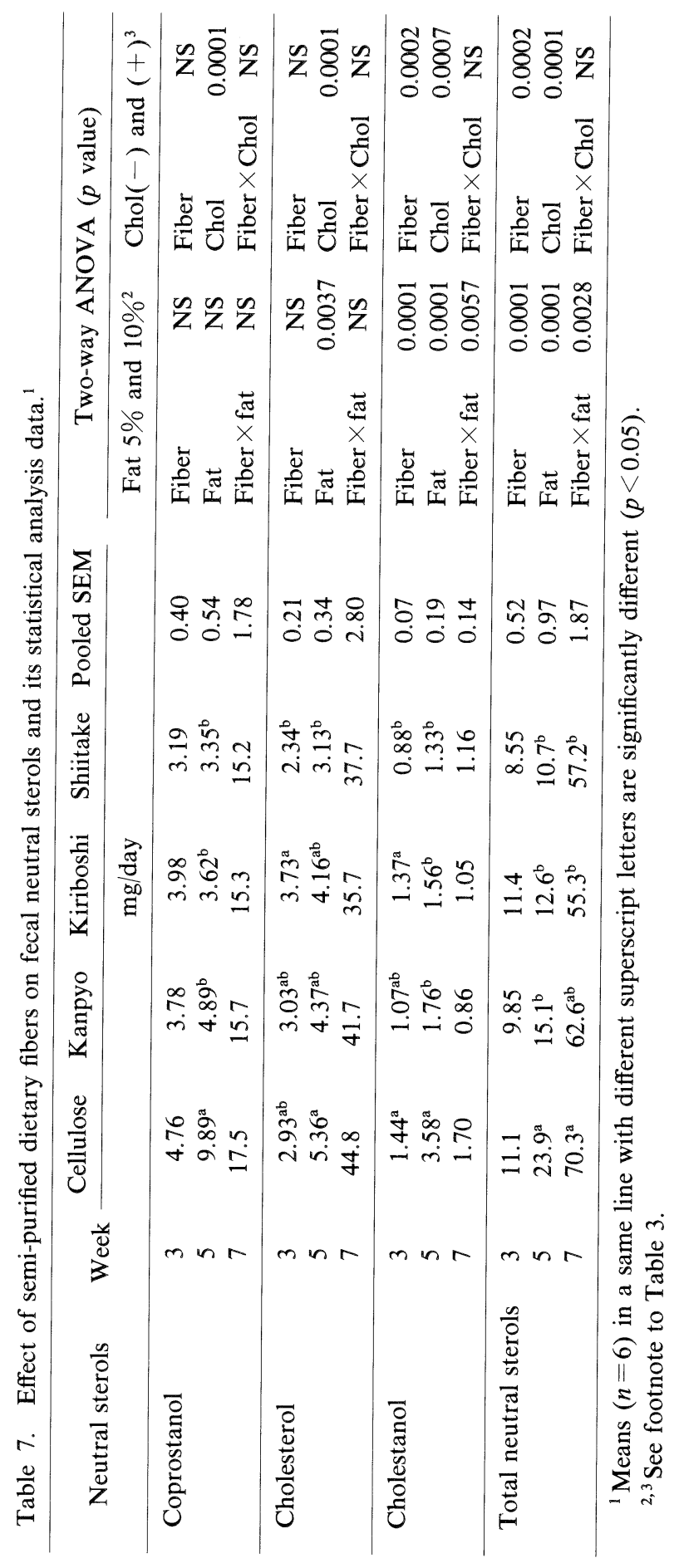


LCA/DCA ratio but no difference was observed among the DF groups. While there was an interaction between fiber and fat, no interaction was seen between fiber and Chol.

Table 7 shows major NS excretions in feces per day. The excretion of cholestanol significantly decreased or showed a decreasing tendency in all the DF groups compared to the $\mathrm{CP}$ group. The excretion increased in all the DF groups when the fat level was elevated. There was an interaction between fiber and fat. Exactly the same tendency was observed in the total NS excretions. Although Chol addition increased the excretion of each NS, the effect of DF was seen only in the excretions of cholestanol and total NS.

\section{DISCUSSION}

Since the same animals were successively used in the experiments, changes in lipid metabolism by aging should be considered the effects of Chol metabolism (28, 29 ) and fecal steroid excretions $(30,31)$. According to Uchida et al. (31), however, such changes occur over a prolonged period of time and any changes taking place in 8 to 12 weeks are minimal, with no changes in serum Chol. We also observed no significant elevations in plasma $\mathrm{Chol}$ from the beginning of the experiment up to the 5 th week (data not included), and therefore judged that the effect of aging, if any, over the 7-week experimental period was very small. We nevertheless minimized such effects as much as possible in the statistical analysis of changes over time.

Fecal wet weight per day in the test DF groups was significantly decreased in any of the measurement weeks when compared to the $\mathrm{CP}$ group. This is presumably because, for one, the test DF levels were different from the CP group, and for another, the DF preparations were more easily decomposed and fermented by intestinal bacteria than cellulose because of their relatively high hemicellulose content. However, significant increases in the fecal water content were observed in both the Kanpyo and Shiitake groups compared to the CP and Kiriboshi groups. The reason for this is not clear but it may be attributed to the difference in their chemical structures.

A larger number of BA showed significance when excretions were evaluated per $1 \mathrm{~g}$ of feces than when evaluated per day. The effect of fecal output is presumably involved in this difference. The fat level had the effect on the fecal dry weight but not on the total BA excretions per day.

Excretions of individual BA differed depending on the type of DF and on the absence/presence of Chol, and showed no clear tendency. There was, however, one noteworthy phenomenon; excretions of LCA, DCA, $\alpha$-MCA, $\beta$-MCA and total BA in the Shiitake group increased significantly or showed an increasing tendency compared to those in the CP group in any week of measurement.

Although the reason for the increase in excretion of these BA in the Shiitake group is not clear at present, it is highly likely that hemicellulose, which is abundant in the Shiitake DF, induced a change in the intestinal environment, which in turn 
produced a change in Chol or BA metabolism. The involvement of eritadenine is assumed to be minimal but cannot be ruled out completely since its content in the DF preparation was not determined.

The ratio of secondary BA to total BA was affected more strongly by the fat level than by the type of DF, and not by Chol addition. Reddy et al. (15) reported that pectin, carrageenan and alfalfa significantly increased total BA excretions compared to cellulose whereas no change was produced by wheat bran and that the proportion of CA metabolites was markedly larger than that of CDCA metabolites only in animals fed carrageenan.

Story (16) reported that the excretions of mainly CDCA derivatives were increased in rats fed DF such as cellulose, oat bran, corn bran and alfalfa. Thomas et al. (32) observed that the proportion of CDCA derivatives was larger than CA in pectin-fed rat feces. According to Vahouny et al. (17), alfalfa, guar gum, psyllium and wheat bran lowered and pectin and mixed fiber raised the CDCA/CA ratio compared to cellulose. In our study, the excretion of CDCA metabolites and CA metabolites both tended to increase. The CDCA/CA ratio was lowered in the test DF groups compared to the CP group.

As discussed above, the fecal excretion of secondary BA was affected by the difference in the type of DF and the results of some experiments on the same type of DF differed among researchers (experimental conditions).

In the present study, the LCA/DCA ratio of feces in the test DF groups was lowered compared to that in the CP group and was affected by the fat level, showing the interaction between fiber and fat. Chol addition produced higher LCA/DCA ratios in all the groups excluding the $\mathrm{CP}$ group, but no difference was observed between the $\mathrm{CP}$ and test DF groups. Gallaher and Franz (21) studied the relation between three kinds of wheat bran and the corn oil level and reported that the fecal LCA/DCA ratio was affected by neither the type of DF nor the fat level. They (22) also studied the relation between the corn oil level and oat bran, rye bran, barley bran and sugar beet fiber, and found that the fiber type affected the LCA/DCA ratio independent of the fat level. It is known that Chol supplementation promotes CDCA metabolism $(18,19)$. It is therefore considered that Chol addition to DF, with its LCA/DCA lowering action, raises this ratio and masks any difference that may exist among DF.

The present study revealed that the LCA/DCA ratio, one of the several indices showing characteristics of fecal BA, responds well to the DF tested. As reported by Gallaher et al. $(21,22)$, this response seems to differ by the type of DF. The significance of the response of the LCA/DCA ratio in evaluating the function of DF needs to be clarified. Further studies are warranted to elucidate the relation between the composition and ratios of fecal steroids and the type of DF in association with colon cancer risks.

We thank Associate Prof. Niebe, A. of the Computer Center, Tokyo University of Agriculture, for giving us kind advice regarding statistical significance analysis of the data. 


\section{REFERENCES}

1) Reddy, B. S., and Wynder, E. L. (1973): Large bowel carcinogenesis: fecal constituents of populations with diverse incidence rates of colon cancer. J. Natl. Cancer Inst., 50, $1437-1442$.

2) Reddy, B. S., and Wynder, E. L. (1977): Metabolic epidemiology of colon cancer: Faecal bile acids and neutral sterols in colon cancer patients and patients with adenomatous polyps. Cancer, 39, 2533-2539.

3) Hill, M. J. (1986): Bile acids and colorectal cancer in humans, in Dietary FiberBasic and Clinical Aspects, ed. by Vahouny, G. V., and Kritchevsky, D., Plenum, New York, pp. 497-513.

4) Chomchai, C., Bhadrachari, N., and Nigro, N. D. (1974): The effect of bile on the induction of experimental intestinal tumors in rats. Dis. Colon Rectum, 17, 310-312.

5) Narisawa, T., Magadia, N., Weisburger, J., and Wynder, E. L. (1974): Promoting effect of bile acids on colon carcinogenesis after intrarectal instillation of $\mathrm{N}$-methyl-Nnitrosoguanidine in rats. J. Natl. Cancer Inst., 53, 1093-1097.

6) Cohen, B. I., Raicht, R. F., Deschner, E. E., Takahashi, M., Sarval, A. M., and Fazzini, E. (1980): Effect of cholic acid feeding on N-methyl-N-nitrosourea induced colon tumors and cell kinetics in rats. J. Natl. Cancer Inst., 64, 573-578.

7) Silverman, S. J., and Andrews, A. W. (1977): Bile acid: co-mutagenic activity in the salmonella-mammalian-microsome mutagenicity test. J. Natl. Cancer Inst., 59, 15571559.

8) Wilpart, M., Mainguet, P., Maskens, A., and Roberfroid, M. (1983): Mutagenicity of 1,2-dimethylhydrazine towards Salmonella typhimurium, co-mutagenic effect of secondary biliary acids. Carcinogenesis, 4, 45-48.

9) Wilpart, M., and Roberfroid, M. (1986): Effects of secondary biliary acids on the mutagenicity of $\mathrm{N}$-methyl- $\mathrm{N}^{\prime}$-nitro-N-nitrosoguanidine, 2-acetylaminofluorene and 2nitrofluorene towards Salmonella typhimurium strains. Carcinogenesis, 7, 703-706.

10) Owen, R. W., Dodo, M., Thompson, M. H., and Hill, M. J. (1984): The faecal ratio of lithocholate to deoxycholate may be an important aetiological factor in colo-rectal cancer. Biochem. Soc. Trans., 12, 861.

11) Owen, R. W., Thompson, M. H., Hill, M. J., Wilpart, M., Mainguet, P., and Roberfroid, M. (1987): Importance of the ratio of lithocholic to deoxycholic acid in large bowel carcinogenesis. Nutr. Cancer, 9, 67-71.

12) Reddy, B. S., Weisburger, J. H., and Wynder, E. L. (1974): Effects of dietary fat level and dimethylhydrazine on fecal acid and neutral sterol excretion and colon carcinogenesis in rats. J. Natl. Cancer Inst., 52, 507-511.

13) Reddy, B. S., Mangat, S., Sheinfil, M. A., Weisburger, J. H., and Wynder, E. L. (1977): Effect of type and amount of dietary fat and 1,2-dimethylhydrazine on biliary bile acids, fecal bile acids, and neutral sterols in rats. Cancer Res., 37, 2132-2137.

14) Sakaguchi, M., Minoura, T., Hiramatsu, Y., Takada, H., Yamamura, M., Hioki, K., and Yamamoto, M. (1986): Effects of dietary saturated and unsaturated fatty acids on fecal bile acids and colon carcinogenesis induced by azoxymethane in rats. Cancer Res., 46, 61-65.

15) Reddy, B. S., Watanabe, K., and Sheinfil, A. (1980): Effect of dietary wheat bran, 
alfalfa, pectin and carrageenan on plasma cholesterol and fecal bile acid and neutral sterol excretion in rats. J. Nutr., 110, 1247-1254.

16) Story, J. A. (1986): Modification of steroid excretion in response to dietary fiber, in Dietary Fiber, Basic and Clinical Aspects, ed. by Vahouny, G. V., and Kritchevsky, D., Plenum, New York, pp. 253-264.

17) Vahouny, G. V., Khalafi, R., Satchithanandam, S., Watkins, D. W., Story, J. A., Cassidy, M. M., and Kritchevsky, D. (1987): Dietary fiber supplementation and fecal bile acids, neutral steroids and divalent cations in rats. J. Nutr., 117, 2009-2015.

18) Beher, W. T., Casazza, K. K., Beher, M. E., Filus, A. M., and Bertasius, J. (1970): Effect of cholesterol on bile acid metabolism in the rat. Proc. Soc. Exp. Biol. Med., 134, 595-602.

19) Uchida, K., Nomura, Y., Kadowaki, M., Takeuchi, N., and Yamamura, Y. (1977): Effect of dietary cholesterol on cholesterol and bile acid metabolism in rats. Jpn. J. Pharmacol., 27, 193-204.

20) Lupton, J. R. (1995): Fat/fiber interactions: Effect on colon physiology and colonic cytokinetics, in Dietary Fiber in Health and Disease, ed. by Kritchevsky, D., and Bonefield, C., Eagan Press, St. Paul, pp. 115-125.

21) Gallaher, D. D., and Franz, P. M. (1990): Effects of corn oil and wheat brans on bile acid metabolism in rats. J. Nutr., 120, 1320-1330.

22) Gallaher, D. D., Locket, P. L., and Gallaher, C. M. (1992): Bile acid metabolism in rats fed two levels of corn oil and brans of oat,rye and barley and sugar beet fiber. $J$. Nutr., 122, 473-481.

23) Lee, S. C., and Hicks, V. A. (1990): Modification of the AOAC total dietary fiber method, in New Developments in Dietary Fiber, ed. by Furda, I., and Brine, C. J., Plenum, New York, pp. 237-244.

24) Southgate, D. A. T. (1969): Determination of carbohydrates in foods. II. Unavailable carbohydrates. J. Sci. Food Agric., 20, 332-335.

25) Zak, B. (1957): Simple rapid microtechnic for serum total cholesterol. Am. J. Clin. Pathol., 27, 583-588.

26) Grundy, S. M., Ahrens, E. H., Miettinen, T. A. (1965): Quantitative isolation and gas liquid chromatographic analysis of total fecal bile acids. J. Lipid Res., 6, 397-410.

27) Riegel, B., Moffett, R. B., McIntosh, A. V. (1955): nor-Desoxycholic acid, in Organic Syntheses. Collective Volume, 3, ed. by Horning, E. C., Allen, C. F. H., Shriner, R. L., Drake, N. L., Smith, L. I., Hamilton, C. S., and Snyder, H. R., John Wiley \& Sons, New York, pp. 234-236.

28) Yamamoto, M., and Yamamura, Y. (1971): Changes of cholesterol metabolism in the aging rat. Atherosclerosis, 13, 365-374.

29) Story, J. A., Tepper, S. A., and Kritchevsky, D. (1976): Age-related changes in the lipid metabolism of Fischer 344 rats. Lipids, 11, 623-627.

30) Hruza, Z., and Zbuzkora, V. (1973): Decrease of excretion of cholesterol during aging. Exp. Gerontol., 8, 29-37.

31) Uchida, K., Nomura, Y., Kadowaki, M., Takase, H., Takano, K., and Takeuchi, N. (1978): Age-related changes in cholesterol and bile acid metabolism in rats. J. Lipid Res., 19, 544-552.

32) Thomas, J. N., Kelly, M. J., and Story, J. A. (1984): Alternation of regression of cholesterol accumulation in ratio by dietary pectin. Br. J. Nutr., 51, 339-345. 